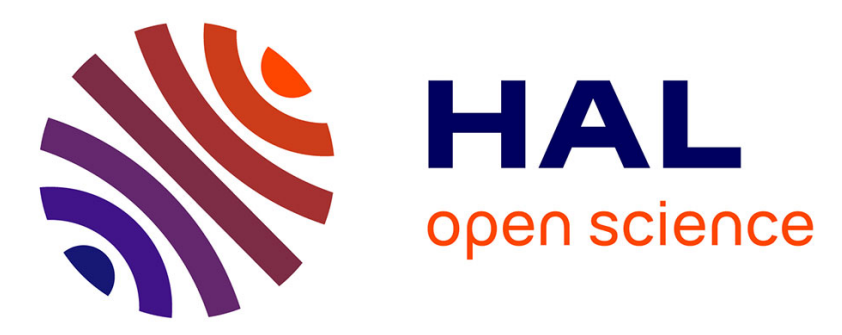

\title{
Shear Wave Elastography can evaluate Annulus Fibrosus Alteration in Adolescent Scoliosis
}

\author{
T Langlais, C Vergari, R Pietton, J Dubousset, W Skalli, R Vialle
}

\section{To cite this version:}

T Langlais, C Vergari, R Pietton, J Dubousset, W Skalli, et al.. Shear Wave Elastography can evaluate Annulus Fibrosus Alteration in Adolescent Scoliosis. European Radiology, 2018, pp.ePub Available. 10.1007/s00330-018-5309-2 . hal-01797569

\section{HAL Id: hal-01797569 \\ https://hal.science/hal-01797569}

Submitted on 22 May 2018

HAL is a multi-disciplinary open access archive for the deposit and dissemination of scientific research documents, whether they are published or not. The documents may come from teaching and research institutions in France or abroad, or from public or private research centers.
L'archive ouverte pluridisciplinaire HAL, est destinée au dépôt et à la diffusion de documents scientifiques de niveau recherche, publiés ou non, émanant des établissements d'enseignement et de recherche français ou étrangers, des laboratoires publics ou privés. 


\title{
Shear Wave Elastography can evaluate Annulus Fibrosus Alteration in Adolescent Scoliosis
}

\author{
Tristan Langlais ${ }^{1,2}$, Claudio Vergari ${ }^{2}$, Raphael Pietton ${ }^{1}$, Jean Dubousset ${ }^{2}$, \\ Wafa Skalli ${ }^{2}$, Raphael Vialle ${ }^{1}$
}

\section{ABSTRACT}

\section{Objectives}

In vitro studies showed that annulus fibrosus lose its integrity in idiopathic scoliosis. Shear wave ultrasound elastography can be used for noninvasive measurement of shear wave speed (SWS) in vivo in the annulus fibrosus, a parameter related to its mechanical properties. The main aim was to assess SWS in lumbar annulus fibrosus of scoliotic adolescents and compare it to healthy subjects.

\section{Methods}

SWS was measured in 180 lumbar IVDs (L3L4, L4L5, L5S1) of thirty healthy adolescents (13 yo \pm 1.9 ) and thirty adolescent idiopathic scoliosis patients (13 yo \pm 2 , Cobb angle: $28.8^{\circ} \pm 10.4^{\circ}$ ). SWS was compared between scoliosis and healthy control group.

\section{Results}

In healthy subjects, average SWS (all disc levels pooled) was $3.0 \pm 0.3 \mathrm{~m} / \mathrm{s}$ whereas, in scoliotic patients it was significantly higher at $3.5 \pm 0.3 \mathrm{~m} / \mathrm{s}(\mathrm{p}=$ 0.0004; Mann-Whitney test). Differences were also significant at all disc levels. No difference was observed between males and females. No correlation was found with age, weight and height.

\section{Conclusion}

Non-invasive shear wave ultrasound is a novel method of assessment to quantitative alteration of annulus fibrosus. These preliminary results are promising to consider shear wave elastography as a biomechanical marker for assessment of idiopathic scoliotic.

1 Department for Innovative Therapies in Musculoskeletal Disease, DHU MAMUTH Department of Pediatric Orthopaedic, Armand Trousseau Hospital, Université Pierre et Marie Curie Paris 6 - 26 avenue du Dr Arnold Netter, 75571 Paris Cedex 12, France

${ }^{2}$ Arts et Metiers ParisTech, LBM/Institut de Biomécanique Humaine Georges Charpak, 151 boulevard de l'hôpital, 75013 Paris, France 


\section{INTRODUCTION}

Adolescent Idiopathic Scoliosis is a three-dimensional spinal deformity of unknown etiology defined in the coronal plane by a Cobb angle of at least $10^{\circ}$ and an axial rotation of the vertebrae [1] . Prevalence varies from 1 to 3\%, pathogenesis and progression risk are not yet fully understood [2-4], although there reasons and risk factors for adolescent scoliosis are proposed [5]. The main challenge is to obtain a better understanding of these factors to establish the appropriate treatment [6-7].

Numerous studies addressed changes of bone structures in scoliotic patients' spine [8-11], but only a few focused on intervertebral disc (IVD), although it probably plays an important role in the vicious cycle leading to curve progression [12]. Recent numerical simulation found a link between progressive scoliosis and a change in IVD mechanical properties [13]. In vitro analysis has shown that annulus fibrosus (AF) can lose its micro-structural integrity in scoliotic specimen at all disc levels [14]. However, existing means to characterize IVD in vivo are either irradiating [15] or expensive, such as magnetic resonance imaging (MRI) [16]. It is commonly accepted that scoliotic spines are stiffer than healthy spines, and that spines with larger deformations are stiffer than those with smaller curves [17]. Nevertheless, this stiffness might also be increased by other structures than the disc (para-spinal musculo-skeletal structures, muscular activation, etc.).

Shear wave elastography is a non-invasive ultrasound-based method that allows quantitative measurement of soft-tissue mechanical properties. It determines the propagation shear wave speed (SWS) in the tissue [18], which is related to the tissue's elastic modulus. Shear wave elastography has been used in clinical practice to evaluate breast [19], liver [20], prostate [21] and muscle [22]. Recently, in vitro and in vivo studies were performed to establish SWS measurement feasibility and reproducibility in IVD or, more precisely, in the disc's outer layer: the annulus fibrosus (AF) [23][24]. Those authors showed correlations between SWS and the mechanical properties of IVD, as well as in vivo reliability both in children and adults. A standardized, reproducible protocol was determined for the acquisition and processing of images for lumbar AF in healthy children and adolescents [25]. Measurements in scoliotic patients, however, are still lacking.

Assessment of disc mechanical properties is of potential clinical interest for several reasons. For instance, disc properties could reveal to be a biomarker of progressive scoliosis at early stage and thus help the physician to adapt the patient's treatment accordingly. Also, the difference of healthy IVD and pathological IVD mechanical properties could guide the decision of lower level instrumentation for spinal fusion.

For this study, it was hypothesized that scoliotic discs should be stiffer than healthy discs and that SWS in the annulus fibrosus could detect this difference.

The main aim of this study was to assess feasibility of disc SWS measurement in scoliotic adolescent patients and to determine the relevance of this technique by comparing SWS to a cohort of healthy subjects.

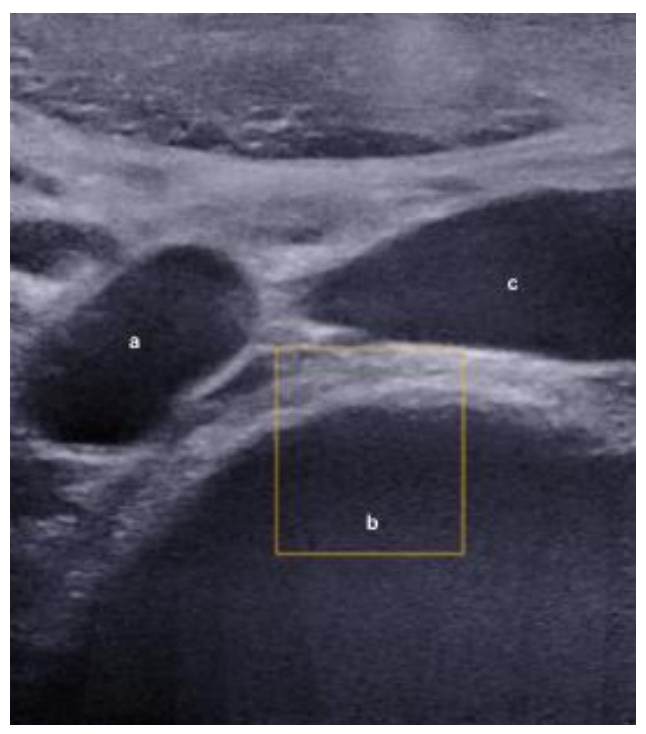

Figure 1: Marking Aortic Iliac Bifurcation. a: aortic bifurcation; b: L4 vertebrae; $c$ : inferior vena cava 


\section{MATERIELS AND METHODS}

\section{Subjects}

Data were collected prospectively from Trousseau university children's hospital within follow-up clinical investigation. Parents and children were informed on the measurement protocol and consented to participating before inclusion. Then parents signed an informed consent, as approved by the ethical committee (C.P.P Île de France IV: Records 14 409).

Children between 10 and 18 years old were included. Healthy children were included in group A if they had no history of spinal disease. A systematic clinical examination was performed by a spine surgeon to rule out any diagnosis of scoliosis for these subjects who were recruited at the trauma consultation. A pathognomonic scoliosis sign, a rib hump and other clinical sign like dorsal pain, asymmetry of the shoulders or pelvic asymmetry were assessed. Patients monitored for fractures of lower limb requiring support discharge were excluded. Scoliotic children were consecutively included in group $\mathrm{S}$ if they had a Cobb angle higher than $10^{\circ}$ and a diagnosis of idiopathic scoliosis. Patients were considered progressive if major curve Cobb angle of more increased more than $6^{\circ}$ between the first and the latest control, [26] and Cobb angle was between $25^{\circ}$ and $50^{\circ}$. Treatment was a Charleston or Caen night time brace and all patients had been treated for a minimum of 3 months. Stable scoliosis was defined by a progression of major curve Cobb angle lower than $6^{\circ}$ between the first and the latest control [26], a Cobb angle lower than $25^{\circ}$ and a Risser stage $\geq 3$ [5]. The first control was the first visit with surgeon at Trousseau hospital whereas the latest control corresponds to the last visit during the inclusion period, i.e. between November 2016 and April 2017. Children with a transitional anomaly, antecedent connective tissue pathology or antecedent spine surgery were excluded from both groups.

Weight, height and body mass index were measured for each subject.

\section{IVD SWS Acquisition Protocol}

SWS was measured in all included subjects following a previously described protocol for lumbar IVD of adolescents [25]. Measurements were performed with an Aixplorer (SuperSonic Imagine, France) and a superlinear S1 10-2 probe. Subjects were in supine position and measurements were performed at disc levels L3-L4, L4-L5 and L5-S1. The level was determined by observing the aortic bifurcation (Figure 1) which, in numerous cases, corresponds to the L4 vertebrae [27]. The aorta or iliac arteries were followed up and down to find the IVDs. IVD was clearly identifiable by the lamellae of the AF (Figure 2). Three clips of about $10 \mathrm{~s}$ (about 10 elastography frames per clip) were recorded for each disc when the signal appeared smooth and stable. A single operator was trained by a radiologist and performed $50+$ SWS disc acquisitions before doing all the measurements for this study. The same operator

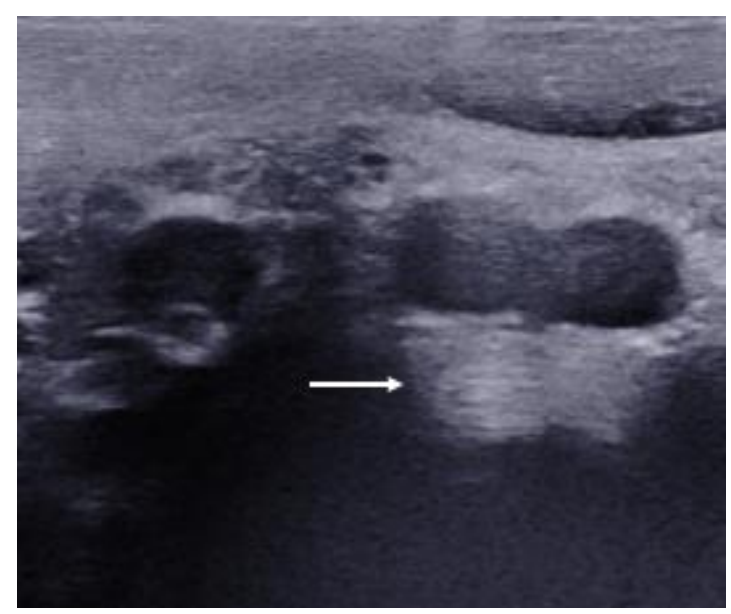

Figure 2: Example of ultrasound of L4-L5 annulus fibrosus. The arrow shows the concentric parallel lamellae. 
also processed the data offline with custom software written in MATLAB (The MathWorks Inc., Natick, MA, USA), as previously detailed [25]. A region of interest (ROI) was manually defined over the AF [23] (Figure 3); the ROI was then semi-automatically tracked in the following images to always measure the same region in all images. The procedure was repeated for the three clips and the average SWS was calculated in each ROI to obtain a single SWS value (from 3 clips x 10 frames $=30$ images per disc) for a given subject and disc level. The average duration of acquisition was 10 minutes for all three discs.

\section{Radiological exam}

Biplanar x-rays (EOS system, EOS imaging, Paris, France) [28] were acquired within normal clinical followup of the scoliotic patients. 3D reconstruction of the spine was performed by a trained operator, which allowed measuring major curve Cobb angle [29]. Risser sign [30] was also

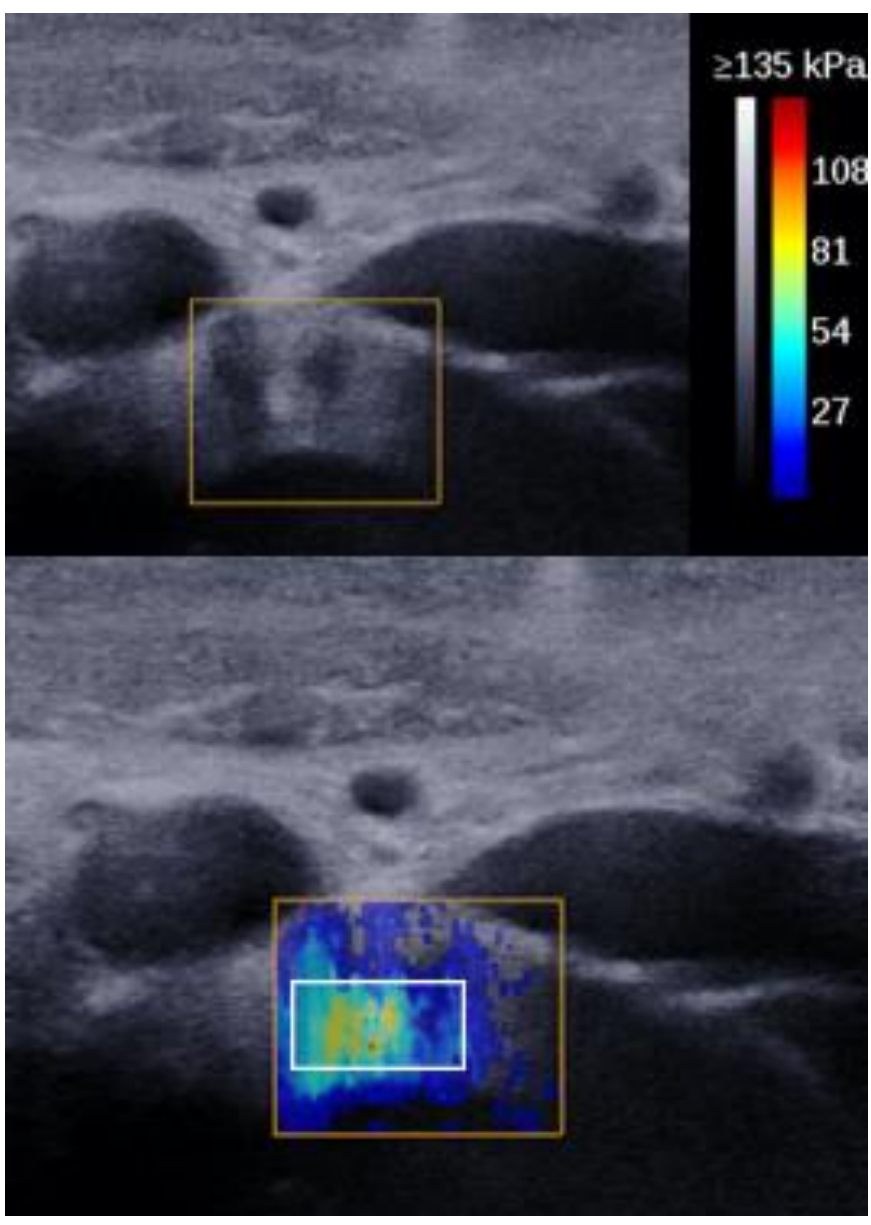

Figure 3: Example of ultrasound (top frame) and elastography image (bottom frame) of L3-L4 annulus fibrosus. The white rectangle is the region of interest (ROI) in the annulus fibrosus.

determined, and the curves were classified according to Scoliosis Research Society recommendations. Junctional vertebral levels were determined by an experimented physician by noting a discontinuity of intervertebral axial rotation and high lateral rotation.

\section{Statistical Analysis}

SWS data was analyzed by disc level and by subject (i.e., pooling the three measured discs for a given subject). A normality corridor of healthy subjects was calculated as the range $\left[5^{\text {th }}-\right.$ $95^{\text {th }}$ percentiles]. Risk ratios were calculated by counting the number of progressive $(\mathrm{P})$ and stable (T) patients having a high (h subscript) or normal/low SWS (n subscript) compared to the normality corridor:

$$
R i s k=\frac{P h /(P h+T h)}{T n /(T n+P n)}
$$

Differences between scoliosis and healthy groups were analyzed with Mann - Withney tests while differences between vertebral levels with Kruskal - Wallis tests. Correlations were quantified with Spearman's rank correlation coefficient. Significance was set at 0.05.

\section{RESULTS}


Sixty adolescents $(13.2 \pm 2$ years old; range 10 to 18 ; 43 girls and 17 boys) were included and analyzed, for a total of 180 lumbar IVDs (3 discs $x \quad 60$ inclusions). Subjects were divided in healthy group (A, $13 \pm$ 1.9 years old; range 10 to $16 ; 19$ girls and 11 boys) and scoliosis group $(\mathrm{S}, 13 \pm 2$ years old; range 10 to $18 ; 24$ girls and 6 boys, average $\mathrm{Cobb}$ angle $28.8^{\circ} \pm$ $\left.10.5^{\circ}\right)$. The characteristics of the subjects are detailed in Table 1. Measurement was not possible in one healthy subject who could not completely relax his belly muscles, thus making it impossible for the probe to get closer to the disc.

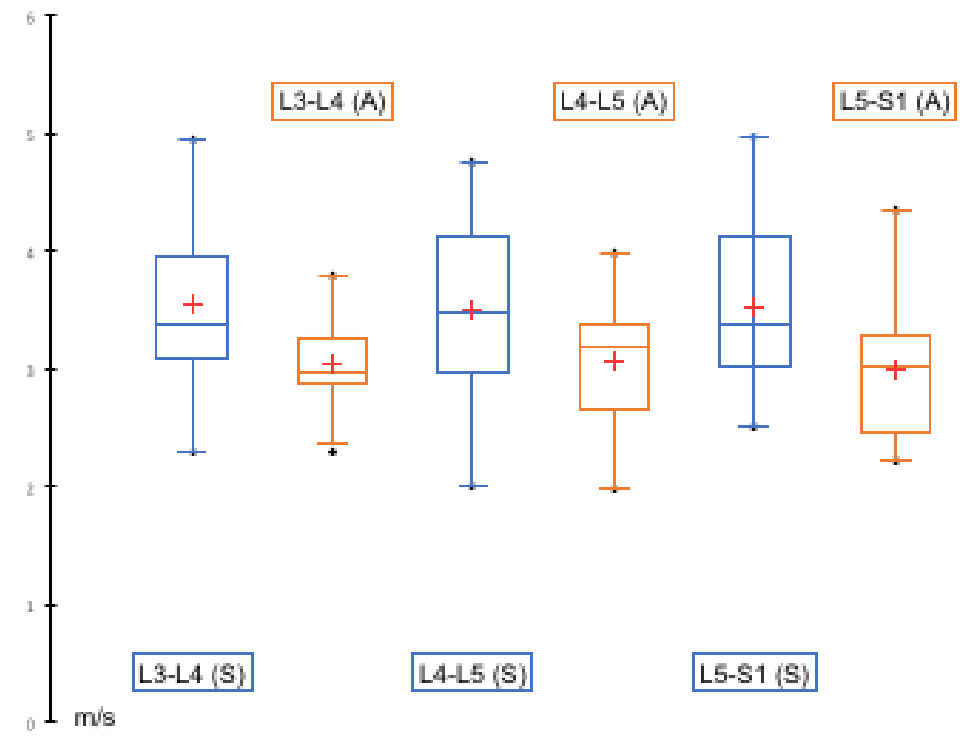

Figure 4: Box plots of average SWS for each level in Scoliotic patient (S) and Healthy adolescent (A). Upper and lower box range represent the upper and lower quartile. Whiskers show the highest and lowest values. The middle line in the box show the median value and the average is represented by the cross. Upper and lower dotted line represent the high and low normality corridor.

\section{Healthy Group SWS}

Average SWS lumbar annulus fibrosus was $3.0 \pm 0.3 \mathrm{~m} / \mathrm{s}$, range 2.3 to $3.7 \mathrm{~m} / \mathrm{s}$; values per level are detailed in Table 2. There was no significant difference between levels L3-L4 (range 2.3 to $3.8 \mathrm{~m} / \mathrm{s}$ ), L4-L5 (range 2.0 to $4.0 \mathrm{~m} / \mathrm{s}$ ) and L5-S1 (range 2.2 to $4.4 \mathrm{~m} / \mathrm{s})(\mathrm{p}=0.7$, Kruskal - Wallis test, Figure 4). 95\% of the healthy subjects had a SWS between a normality corridor of 2.3 and $3.8 \mathrm{~m} / \mathrm{s}$. The difference in average SWS between girls $(3.1 \pm 0.4 \mathrm{~m} / \mathrm{s})$ and boys $(2.9 \pm 0.2 \mathrm{~m} / \mathrm{s})$ was not significant $(\mathrm{p}=0.2$, Mann - Whitney test $)$. No correlation of SWS was found with age, weight, height or BMI ( $p>0.05$, Spearman's test).

\section{Scoliosis Group SWS}

Thirty idiopathic scoliotic patients $(13 \pm 2$ years old; range 10 to $18 ; 24$ girls and 6 boys, average Cobb angle $28.8^{\circ} \pm 10.5^{\circ}$ ) were included. Among these children, there were twenty progressive scoliosis at pre-treatment stage (10 patients; $13 \pm 2$ years old, range 11 to 16 ; 8 girls and 2 boys; average Cobb angle : $30,8^{\circ} \pm 9,3^{\circ}$, range $26,6-49,5^{\circ}$ ) or during treatment (10 patients; $13 \pm 2.4$ years old; range 10 to $16 ; 8$ girls and 2 boys; average Cobb angle : $36,5^{\circ}$ $\pm 8,4^{\circ}$, range $\left.25-48,6^{\circ}\right)$ and ten stable scoliosis which were never treated ( $14 \pm 1.5$ years old; range 13 to $18 ; 8$ girls and 2 boys; average Cobb angle : $19.1^{\circ} \pm 4,1^{\circ}$, range $12.7-25^{\circ}$ ). All types of curves were represented and they were classified according to Scoliosis Research Society recommendations. The characteristics of the subjects are detailed in Table 1.

Average SWS lumbar annulus fibrosus was $3.5 \pm 0.3 \mathrm{~m} / \mathrm{s}$, range 2.7 to $4.8 \mathrm{~m} / \mathrm{s}$; SWS per disc level are detailed in Table 2. There was no significant difference between levels L3-L4 (range 2.3 to $4.9 \mathrm{~m} / \mathrm{s}$ ), L4-L5 (range 2.0 to $4.7 \mathrm{~m} / \mathrm{s}$ ) and L5-S1 (range 2.5 to $5.0 \mathrm{~m} / \mathrm{s}, \mathrm{p}=0.98$, Kruskal - Wallis test, Figure 4). 
There was a weak correlation between average SWS and BMI in the scoliosis group $(\mathrm{p}=$ 0.04 , Spearman's rho $=0.4)$. No correlation of SWS was found with age, weight, height or Cobb angle ( $p>0.05$, Spearman's test), nor any effect of Risser sign $(p>0.05)$.

Average SWS in stable scoliosis was $3.3 \pm 0.3 \mathrm{~m} / \mathrm{s}$, range 2.7 to $4.3 \mathrm{~m} / \mathrm{s}$ whereas in progressive scoliosis average SWS was $3.7 \pm 0.3 \mathrm{~m} / \mathrm{s}$, range 2.8 to $4.8 \mathrm{~m} / \mathrm{s}$ ( $\mathrm{p}=0.06$, Mann Whitney test). In progressive scoliosis at pre -treatment stage SWS mean was $4.0 \pm 0.3 \mathrm{~m} / \mathrm{s}$, range 3.0 to $4.7 \mathrm{~m} / \mathrm{s}$ while in progressive scoliosis during treatment SWS mean was significantly lower at $3.3 \pm 0.3 \mathrm{~m} / \mathrm{s}$, range 2.8 to 3.7 $\mathrm{m} / \mathrm{s}(\mathrm{p}=0.01$, Mann - Whitney test).

\section{Groups comparison}

SWS was significantly higher in scoliotic patients that in healthy subjects at all disc levels ( $\mathrm{p} \leq 0.02$ for all levels) and when all levels were pooled ( $\mathrm{p}<0.001$, Mann - Whitney test) (Figure 4 and Table 2). Figure 4 also shows higher SWS variability in scoliosis group. No significant difference was observed between males and females at any level (nor with all levels pooled, $p>0.05$ ). For the whole population pooled, no correlation of average SWS was found with age, weight and height.

One AF out of ninety presented a SWS lower than the previously defined normality corridor, while 29 AF (32\%) from sixteen patients had a SWS higher than the normality corridor. These abnormal values were evenly spread among all three vertebral levels, and they were not concentrated in specific patients, i.e., only five patients had all three discs characterized by an abnormally high SWS. These five patients had an average Cobb angle of $32.1^{\circ}$, so not much different from the population's average, but they were all characterized by a progressive scoliosis.

Of the sixteen patients with

\begin{tabular}{|c|c|c|}
\hline \multicolumn{3}{|c|}{ Table 1 : Characteristics of Subjects } \\
\hline & $\begin{array}{l}\text { Healthy } \\
\text { Subjects }\end{array}$ & $\begin{array}{l}\text { Scoliotic } \\
\text { Subjects }\end{array}$ \\
\hline & $\mathrm{n}=30$ & $\mathrm{n}=30$ \\
\hline \multicolumn{3}{|l|}{ Age (yo) } \\
\hline Range & $10-16$ & $10-18$ \\
\hline Mean \pm SD & $13 \pm 1,9$ & $13 \pm 2$ \\
\hline \multicolumn{3}{|l|}{ Gender } \\
\hline Boys & 11 & 6 \\
\hline Girls & 19 & 24 \\
\hline \multicolumn{3}{|l|}{ Height (cm) } \\
\hline Range & $1,3-1,8$ & $1,3-1,8$ \\
\hline Mean \pm SD & $1,6 \pm 0,1$ & $1,6 \pm 0,1$ \\
\hline \multicolumn{3}{|l|}{ Weight (kg) } \\
\hline Range & $35-73$ & $21-65$ \\
\hline Mean \pm SD & $48,4 \pm 11,8$ & $45,8 \pm 9,4$ \\
\hline \multicolumn{3}{|l|}{ B.M.I } \\
\hline Range & $12,6-27,6$ & $13,3-23$ \\
\hline Mean \pm SD & $19 \pm 3,2$ & $18,1 \pm 2,6$ \\
\hline \multicolumn{3}{|l|}{ Risser } \\
\hline$\leq 2$ & & 12 \\
\hline$\geq 3$ & & 18 \\
\hline \multicolumn{3}{|l|}{$\begin{array}{ll}\text { Cobb } & \text { Angle } \\
\text { Major Curve }\left(^{\circ}\right) & \end{array}$} \\
\hline Range & & $12,7-49,5$ \\
\hline Mean \pm SD & & $28,8 \pm 10,4$ \\
\hline \multicolumn{3}{|l|}{ Types of Curves } \\
\hline Main thoracic & & 6 \\
\hline Main Thoracolumbar & & 7 \\
\hline Main Lumbar & & 6 \\
\hline Double major & & 7 \\
\hline Triple major & & 4 \\
\hline
\end{tabular}


abnormally high SWS, $60 \%$ belonged to the progressive scoliosis group while $40 \%$ to the stable group. This corresponds to a risk ratio of 1, given the different cohort sizes of stable and progressive groups; therefore, high SWS was not associated with increased risk of curve progression, unless several discs had high SWS.

Lower junctional levels were within the three measured disc levels in six patients of the progressive scoliosis group and nine patients of the stable group. In progressive scoliosis, five out of six SWS values $(83 \%)$ were above the normality corridor whereas only two out of nine SWS values $(22 \%)$ of were higher than normality corridor in stable scoliosis. This corresponds to a risk ratio of 4.6. In other words, abnormally high SWS in lumbar discs does not necessarily correspond to an increased risk of progression, unless several discs or a junctional disc show abnormally high SWS.

Table 2: Differences in SWS

Scoliotic vs Asymptomatic; MannWhitney test:

\begin{tabular}{|l|l|}
\hline Level & P - Value \\
\hline All levels & 0.00039 \\
\hline L3-L4 & 0.0022 \\
\hline L4-L5 & 0.02 \\
\hline L5-S1 & 0.0039 \\
\hline
\end{tabular}

\section{DISCUSSION}

Results of the present work showed higher SWS in scoliotic lumbar disc than in healthy subjects in L3-L4, L4-L5 and L5-S1. These results confirm that there are biomechanical changes in AF in scoliotic patients. Moreover, SWS increases with the tissue's elastic modulus, suggesting that scoliotic discs are stiffer than healthy ones, as initially hypothesized. It was also hypothesized that SWS should increase with deformation severity, but no correlation was observed between SWS and Cobb angle. Huber et al. [31] reported that degeneration of IVD is greater within the curvature than in healthy areas, so it is possible that this lack of correlation, in our study, is due to the pooling of different spinal topologies.

The present work represents the first application of shear wave elastography to characterize the mechanical properties of lumbar disc in scoliotic patients. This work stemmed from the conception that scoliotic spines are stiffer than healthy ones; therefore, disc stiffness might represent a biomarker related to scoliosis. Although MRI elastography has allowed to quantify the mechanical properties of IVD in vitro [32][33] and in vivo [16] , ultrasound elastography seemed a good candidate for routine use in clinical setting. Indeed, SWS measure is a simple, fast, accessible, reproducible [25] and non-invasive method of IVD assessment with a fast learning curve. Given the heterogeneous character of the medium crossed by the ultrasound waves (i.e., the intestines) and the relative depth of the SWS measurement (about 2-3 cm), the resulting SWS maps appear noisy and contain artefacts (Figure 3). Repeated measurements (30 images per disc in the present study) are necessary to guarantee an inter-operator reproducibility of $8.7 \%$ previously obtained in vivo [25]. The measurement is fast so this does not significantly impact the examination time, and the image processing can be performed afterwards. To obtain a clear image, the operator should not hesitate to replace the probe several times and apply constant pressure to move the intestines and their contents. The probe should also be carefully aligned with the IVD plane. Depth of measurement could have an effect on the observed SWS. While this parameter was not controlled, the probe can usually be pushed to $2-3 \mathrm{~cm}$ from the disc, even in older subjects (i.e. 18 years old). Besides, the previously cited reproducibility values were obtained without controlling for depth either. 
IVD is composed of three regions: annulus fibrosus, the transition zone and nucleus pulposus [34]. AF is organized in concentric arrays of parallel lamellae composed of collagen and elastin [34]. This elastic fiber networks becomes denser with aging [34]. Yu et al. [13] reported that in scoliotic patients the elastic fibers were sparse and the collagen and elastic fiber networks were disorganized and loss of lamellar structure. Kobielarz et al.[35] confirmed this alteration and reported that the number of elastin fiber and collagen I were reduced in AF. In vivo, Huber et al. [31] showed by MRI that IVD were more degenerated in scoliosis than healthy IVD whereas Schlösser et al. found by CT scan analysis that deformation in 3-D was greater in the IVD than in the vertebral bodies [15].

Shear wave elastography could be an aid for the detection of progressive scoliosis. SWS was lower for the stable scoliosis group than for the progressive scoliosis. Moreover, SWS at junctional level was in $83 \%$ discs higher than the normal corridor in progressive scoliosis, with an increased risk ratio of 4.6 relative to stable scoliosis. Although these results should be confirmed on a larger cohort, they are very promising for the early detection of progressive patients. Recently, a severity index (S-index) consisting of a combination of quantitative 3D geometrical parameters was proposed to estimate the risk of scoliosis progression at early stage [5]. The initial results showed a sensitivity of $89 \%$ and a specificity of $84 \%$ when detecting progressive and stable scoliosis. Four of the six geometrical parameters utilized in that work are related to the axial plane: curve torsion, axial rotation of the apical vertebra and inter-vertebral axial rotation at the two junctional vertebral levels. This underlines the importance of the disc and of its torsion in the progression of scoliosis, thus further justifying the need of biomechanical IVD characterization.

Shear wave elastography could also be an aid for the surgical planning. In preoperative, sidebending radiographic images are commonly used to classify the curves according to their topology and flexibility, and to help choosing the vertebral level for instrumentation [36]. In a structural lumbar curve, the choice of the lower level remains debated and not consensual despite various factors like last touch vertebrae or neutral vertebrae [37] or the first mobile IVD on side bending images [36]. SWS measurement could be one more factor for distinguishing the first healthy lumbar IVD and the pathological lumbar IVD. In the present work, SWS mean was lower in progressive scoliosis during treatment than progressive scoliosis at pre-treatment stage. The treatment by brace can induce structural changes of the IVD [38] following to the desired correction. But no study allowed quantifying changes in biomechanical properties in IVD after brace treatment. These results should be validated on a larger cohort.

SWSs in the healthy group were close to results found in a previous previously study on a different cohort [25]. The average SWS reported was $2.9 \pm 0.5 \mathrm{~m} / \mathrm{s}$ (range 1.8 to $3.9 \mathrm{~m} / \mathrm{s}$ ) in 31 healthy children aged $11 \pm 3$ years old, against $3.0 \pm 0.3 \mathrm{~m} / \mathrm{s}$ in the present work. As in the present results, those authors did not find any significant differences between girls and boys or any correlation of SWS with subjects' age, weight or height. It can be noticed that measurement in the present study were performed on more levels than L3-L4.

This study had some limitations: first, a larger cohort is needed to assess the impact of the brace on IVD SWS and to carry out an analysis according to the different types of spine morphology. Second, the operator only has access to the last three or four mobile IVDs and to the anterior part of annulus fibrosus. SWS could not be measured in the thoracic IVDs and measurement position was not controlled relative to the concave or convex part of the curvature. Also, the anatomical landmark used to detect the vertebral level, i.e. the aortic bifurcation, corresponds to the L4 vertebra only in $64 \%$ of the subjects, thus introducing a degree of uncertainty on the level being measured. Nevertheless, having access to the 
adolescent's frontal radiography can help recognize the disc level by comparing lumbar spinal topology with the position of the vertebrae as observed during ultrasound examination. Third, they were no radiological confirmation of absence of scoliosis or absence of transitional anatomy in control subjects. Nevertheless, for those patients that had lumbar curves, a lateral displacement of the spine relative to the abdominal wall was clearly visible (Figure 1). Finally, measurement inter-operator reproducibility was not tested in this study, although the same protocol was used from previous work where measurement reliability was determined.

Despite these limitations, non-invasive shear wave ultrasound elastography proved to be a feasible, fast, and reliable means of quantifying disc mechanical properties, and, most of all, it resulted sensitive to the biomechanical alteration of $\mathrm{AF}$ in scoliotic patients. In particular, results suggest that a junctional vertebra with high SWS could be associated with an increased risk of scoliosis progression. Therefore, ultrasound elastography has the potential of being a novel early biomarker of progressive scoliosis, and it could be included in routine use in clinical setting since it is not invasive, fast and accessible. These preliminary results open the way for larger studies aiming at confirming these findings.

\section{REFERENCES}

1. Coonrad RW, Murrell GA, Motley G, et al (1998) A logical coronal pattern classification of 2,000 consecutive idiopathic scoliosis cases based on the scoliosis research society-defined apical vertebra. Spine 23:1380-1391.

2. Lonstein JE (1994) Adolescent idiopathic scoliosis. Lancet Lond Engl 344:1407-1412.

3. Kouwenhoven J-WM, Castelein RM (2008) The pathogenesis of adolescent idiopathic scoliosis: review of the literature. Spine 33:2898-2908. doi: 10.1097/BRS.0b013e3181891751

4. Altaf F, Gibson A, Dannawi Z, Noordeen H (2013) Adolescent idiopathic scoliosis. BMJ 346:f2508.

5. Skalli W, Vergari C, Ebermeyer E, et al (2016) Early Detection of Progressive Adolescent Idiopathic Scoliosis: A Severity Index. Spine. doi: 10.1097/BRS.0000000000001961

6. Duval-Beaupère G (1992) Rib hump and supine angle as prognostic factors for mild scoliosis. Spine 17:103-107.

7. Duval-Beaupere G (1996) Threshold values for supine and standing Cobb angles and rib hump measurements: prognostic factors for scoliosis. Eur Spine J Off Publ Eur Spine Soc Eur Spinal Deform Soc Eur Sect Cerv Spine Res Soc 5:79-84.

8. Shi L, Wang D, Driscoll M, et al (2011) Biomechanical analysis and modeling of different vertebral growth patterns in adolescent idiopathic scoliosis and healthy subjects. Scoliosis 6:11. doi: 10.1186/1748-7161-6-11

9. Nault M-L, Mac-Thiong J-M, Roy-Beaudry M, et al (2014) Three-dimensional spinal morphology can differentiate between progressive and nonprogressive patients with adolescent idiopathic scoliosis at the initial presentation: a prospective study. Spine 39:E601-606. doi: 10.1097/BRS.0000000000000284

10. Nault M-L, Mac-Thiong J-M, Roy-Beaudry M, et al (2014) Three-Dimensional Spinal Morphology can Differentiate Between Progressive and Non-Progressive Patients With Adolescent Idiopathic Scoliosis at the Initial Presentation. Spine. doi: 10.1097/BRS.0000000000000284 
11. Modi HN, Suh SW, Song H-R, et al (2008) Differential wedging of vertebral body and intervertebral disc in thoracic and lumbar spine in adolescent idiopathic scoliosis - A cross sectional study in 150 patients. Scoliosis 3:11. doi: 10.1186/1748-7161-3-11

12. Stokes IA, Spence H, Aronsson DD, Kilmer N (1996) Mechanical modulation of vertebral body growth. Implications for scoliosis progression. Spine 21:1162-1167.

13. Drevelle $\mathrm{X}$, Lafon $\mathrm{Y}$, Ebermeyer E, et al (2010) Analysis of idiopathic scoliosis progression by using numerical simulation. Spine 35:E407-412. doi: 10.1097/BRS.0b013e3181cb46d6

14. Yu J, Fairbank JCT, Roberts S, Urban JPG (2005) The elastic fiber network of the anulus fibrosus of the normal and scoliotic human intervertebral disc. Spine 30:1815-1820.

15. Schlösser TPC, van Stralen M, Brink RC, et al (2014) Three-dimensional characterization of torsion and asymmetry of the intervertebral discs versus vertebral bodies in adolescent idiopathic scoliosis. Spine 39:E1159-1166. doi: 10.1097/BRS.0000000000000467

16. Walter BA, Mageswaran P, Mo X, et al (2017) MR Elastography-derived Stiffness: A Biomarker for Intervertebral Disc Degeneration. Radiology 162287. doi: 10.1148/radiol.2017162287

17. Deviren V, Berven S, Kleinstueck F, et al (2002) Predictors of flexibility and pain patterns in thoracolumbar and lumbar idiopathic scoliosis. Spine 27:2346-2349. doi: 10.1097/01.BRS.0000030300.45392.BF

18. Gennisson J-L, Deffieux T, Fink M, Tanter M (2013) Ultrasound elastography: principles and techniques. Diagn Interv Imaging 94:487-495. doi: 10.1016/j.diii.2013.01.022

19. Cong R, Li J, Guo S (2017) A new qualitative pattern classification of shear wave elastograghy for solid breast mass evaluation. Eur J Radiol 87:111-119. doi: 10.1016/j.ejrad.2016.12.021

20. Yoon HM, Kim SY, Kim KM, et al (2017) Liver Stiffness Measured by Shear-wave Elastography for Evaluating intra-hepatic Portal Hypertension in Children. J Pediatr Gastroenterol Nutr. doi: 10.1097/MPG.0000000000001517

21. Rouvière O, Melodelima C, Hoang Dinh A, et al (2016) Stiffness of benign and malignant prostate tissue measured by shear-wave elastography: a preliminary study. Eur Radiol. doi: $10.1007 / \mathrm{s} 00330-016-4534-9$

22. Moreau B, Vergari C, Gad H, et al (2016) Non-invasive assessment of human multifidus muscle stiffness using ultrasound shear wave elastography: A feasibility study. Proc Inst Mech Eng $[\mathrm{H}]$ 230:809-814. doi: 10.1177/0954411916656022

23. Vergari C, Rouch P, Dubois G, et al (2014) Intervertebral disc characterization by shear wave elastography: An in vitro preliminary study. Proc Inst Mech Eng [H] 228:607-615. doi: $10.1177 / 0954411914540279$

24. Vergari C, Rouch P, Dubois G, et al (2014) Non-invasive biomechanical characterization of intervertebral discs by shear wave ultrasound elastography: a feasibility study. Eur Radiol 24:3210-3216. doi: 10.1007/s00330-014-3382-8

25. Vergari C, Dubois G, Vialle R, et al (2015) Lumbar annulus fibrosus biomechanical characterization in healthy children by ultrasound shear wave elastography. Eur Radiol. doi: $10.1007 / \mathrm{s} 00330-015-3911-0$

26. Pruijs JE, Hageman MA, Keessen W, et al (1994) Variation in Cobb angle measurements in scoliosis. Skeletal Radiol 23:517-520.

27. Deswal A, Tamang BK, Bala A (2014) Study of aortic- common iliac bifurcation and its clinical significance. J Clin Diagn Res JCDR 8:AC06-08. doi: 10.7860/JCDR/2014/8767.4559 
28. Dubousset J, Charpak G, Dorion I, et al. (2005) A new 2D and 3D imaging approach to musculoskeletal physiology and pathology with low-dose radiation and the standing position: the EOS system. Bulletin de l'Academie nationale de medecine 189:287-297.

29. Humbert L, De Guise JA, Aubert B, et al (2009) 3D reconstruction of the spine from biplanar $X$ - rays using parametric models based on transversal and longitudinal inferences. Med Eng Phys 31:681-687. doi: 10.1016/j.medengphy.2009.01.003

30. Risser JC (1958) The lliac apophysis; an invaluable sign in the management of scoliosis. Clin Orthop 11:111-119.

31. Huber M, Gilbert G, Roy J, et al (2016) Sensitivity of MRI parameters within intervertebral discs to the severity of adolescent idiopathic scoliosis. J Magn Reson Imaging JMRI 44:1123-1131. doi: $10.1002 /$ jmri.25260

32. Cortes DH, Magland JF, Wright AC, Elliott DM (2014) The shear modulus of the nucleus pulposus measured using magnetic resonance elastography: a potential biomarker for intervertebral disc degeneration. Magn Reson Med 72:211-219. doi: 10.1002/mrm.24895

33. Ben-Abraham El, Chen J, Felmlee JP, et al (2015) Feasibility of MR elastography of the intervertebral disc. Magn Reson Imaging. doi: 10.1016/j.mri.2015.12.037

34. Yu J, Winlove PC, Roberts S, Urban JPG (2002) Elastic fibre organization in the intervertebral discs of the bovine tail. J Anat 201:465-475.

35. Kobielarz M, Szotek S, Głowacki M, et al (2016) Qualitative and quantitative assessment of collagen and elastin in annulus fibrosus of the physiologic and scoliotic intervertebral discs. $J$ Mech Behav Biomed Mater 62:45-56. doi: 10.1016/j.jmbbm.2016.04.033

36. Hirsch C, Ilharreborde B, Mazda K (2016) Flexibility analysis in adolescent idiopathic scoliosis on side-bending images using the EOS imaging system. Orthop Traumatol Surg Res OTSR 102:495-500. doi: 10.1016/j.otsr.2016.01.021

37. Ilharreborde B, Ferrero E, Angelliaume A, et al (2017) Selective versus hyperselective posterior fusions in Lenke 5 adolescent idiopathic scoliosis: comparison of radiological and clinical outcomes. Eur Spine J Off Publ Eur Spine Soc Eur Spinal Deform Soc Eur Sect Cerv Spine Res Soc. doi: 10.1007/s00586-017-5070-2

38. Perie D, De Gauzy JS, Sevely A, Hobatho MC (2002) CTM brace effect on scoliotic intervertebral discs using MRI method. Stud Health Technol Inform 88:230-234. 